\title{
A efetividade do laser de HeNe 632,8 nm no reestabelecimento da integridade dos tecidos cutâneos em animais experimentais: revisão sistemática
}

\author{
Effectiveness of HeNe $632.8 \mathrm{~nm}$ laser on skin tissue integrity repair in experimental \\ animals: a systematic review
}

Clarissa Tôrres Leal', Andrezza de Lemos Bezerra², Andréa Lemos ${ }^{3}$

\begin{abstract}
RESUMO I O objetivo desta revisão sistemática foi analisar o efeito do laser de HeNe na cicatrização de feridas em ratos. Foram selecionados estudos experimentais que adotaram o laser HeNe para o tratamento de feridas agudas em ratos adultos saudáveis, com lesões induzidas por bisturi, nas bases de dados PubMed/MEDLINE, LILACS e SciELO. Foram utilizados os seguintes descritores: cicatrização de feridas e colágeno, de acordo com o MeSH e o DeCS, além dos unitermos laser HeNe e reparação da pele e seus equivalentes em inglês e espanhol. Três estudos foram incluídos na revisão sistemática, não sendo possível a realização de metanálise, devido à impossibilidade de comparação entre as metodologias dos estudos selecionados. Todos os estudos realizaram análise por meio de cortes histológicos das cicatrizes. A presença de fallhas metodológicas nos três artigos dificultou a interpretação fidedigna dos dados encontrados. Os estudos destacaram uma redução na intensidade da resposta inflamatória e uma melhor organização das fibras colágenas no grupo irradiado. A terapia com laser HeNe mostrou boa resposta no reparo tecidual. No entanto, tais resultados devem ser analisados de modo criterioso, uma vez que há presença de heterogeneidade, principalmente em relação aos parâmetros adotados.
\end{abstract}

Descritores I terapia a laser de baixa intensidade; pele; cicatrização de feridas; colágeno.

\begin{abstract}
I The aim of this systematic review was to analyze the effect of HeNe laser on wound healing in rats. Experimental studies that adopted HeNe laser as a treatment of acute lesions in healthy adult rats were selected, with scalpel-induced lesions, at PubMed/ MEDLINE, LILACS, and SCIELO databases. The following keywords were used: wound healing and collagen, according to MeSH and DeCS, besides the terms HeNe laser and skin repair, and their equivalent in Portuguese and Spanish. Three studies were included in this systematic review, but it was not possible to perform a metanalysis, due to impossibility to compare methods of the selected studies. All studies carried out analysis through histological cuts of the scars. The presence of methodological flaws in the three articles hampered reliable interpretation of the findings. The papers highlighted a decrease of the inflammatory response intensity and a greater organization of collagen fibers on the irradiated group. HeNe laser therapy showed a good response on tissue repair. However, these results must be analyzed carefully, since there was heterogeneity, mostly related to the adopted parameters.
\end{abstract}

Keywords I laser therapy, low level; skin; wound healing; collagen.

\footnotetext{
Estudo desenvolvido no Instituto de Medicina Integral Professor Fernando Figueira (IMIP) - Recife (PE), Brasil. 'Especialista em Fisioterapia Dermatofuncional e Cosmetologia pela Faculdade Redentor - Rio de Janeiro (RJ); Fisioterapeuta do Ambulatório de Fisioterapia Dermatofuncional do IMIP; Coordenadora de Tutor do Curso de Fisioterapia da Faculdade Pernambucana de Saúde (FPS) - Recife (PE), Brasil.

${ }^{2}$ Doutoranda em Neuropsiquiatria e Ciências do Comportamento pela Universidade Federal de Pernambuco (UFPE); Fisioterapeuta do Hospital Agamenon Magalhães; Coordenadora de Tutor do Curso de Fisioterapia da FPS - Recife (PE), Brasil.

${ }^{3}$ Doutora em Saúde Materno-infantil pelo IMIP; Docente do Departamento de Fisioterapia da UFPE - Recife (PE), Brasil.
} 


\section{INTRODUÇÃO}

O processo de reparo tecidual é objeto de estudo há anos, devido à alta morbidade relacionada às alterações no curso natural da cicatrização. Percebe-se uma crescente contribuição multidisciplinar para este dinâmico fenômeno fisiológico, que continua desafiando os pesquisadores ${ }^{1-3}$.

Logo após o surgimento de uma lesão, há uma complexa liberação de mediadores que dão início ao processo de reparo, o qual se inicia pela inflamação, fase da migração de leucócitos e plaquetas; seguido pela fase proliferativa ou granulação, com destaque para a angiogênese e para o aumento no número de fibroblastos para produção de colágeno, elastina, fibronectina, glicosaminoglicanos e proteases que remodelam fisiologicamente a ferida; e finalmente, concluí-se com a epitelização ${ }^{4-7}$. Esta última fase dura meses e tem como meta a melhoria nos componentes das fibras colágenas e a reabsorção de água para aumentar a força da cicatriz e diminuir sua espessura. A neovascularização diminui e tardiamente a cicatriz é considerada avascular, assim como relativamente acelular ${ }^{5}$.

Sabe-se que as dificuldades no processo de reparo tecidual ocorrem nos estágios iniciais, nos quais se observa uma acentuação do edema, uma diminuída proliferação vascular e uma redução significativa de elementos celulares como leucócitos, macrófagos e fibroblastos ${ }^{2-4}$. Um estudo recente sugeriu que a instituição de uma intervenção é mais apropriada na fase inicial do processo de reparo tecidual ${ }^{1}$.

Estudos têm demonstrado o papel dos recursos físicos no processo de reparo tecidual e na cicatrização de feridas, no sentido de acelerá-lo. Dentre os vários tratamentos disponíveis, podem ser citados microcorrentes, light emitting diode (LED), ultrassom e terapia com laser ${ }^{8}$.

A terapia com laser (light amplification by stimulated emission of radiation - amplificação da luz por emissão estimulada de radiação) utiliza luz altamente organizada para estimular alterações fisiológicas. É considerada uma terapia não invasiva, indolor, não térmica e asséptica, sem efeitos colaterais, com boa relação custo-benefício, que, aliado à segurança do tratamento, justificam o crescente interesse dos pesquisadores quanto ao mecanismo de ação e seus efeitos terapêuticos ${ }^{9}$.

Os lasers em baixa intensidade ou em fotobiomodulação são agentes terapêuticos sem potencial destrutivo, com eficiente ação anti-inflamatória e analgésica, fatores esses que ajudam no reparo dos tecidos biológicos traumatizados ${ }^{10,11}$. Também atuam na cicatrização pelo aumento do metabolismo, da proliferação e da maturação celular, aumento da quantidade de tecido de granulação e diminuição dos mediadores inflamatórios ${ }^{12}$. A camada tecidual a ser atingida depende do tipo de laser, da potência, do comprimento de onda e do tempo de irradiação. O laser hélio-neônio (HeNe), cujo comprimento de onda é de $632,8 \mathrm{~nm}$, atinge somente as estruturas superficiais e é melhor absorvido pelos tecidos vermelhos ${ }^{4,12}$. Tem aplicações mais frequentes para o tratamento de lesões superficiais como as lesões estéticas ou em processo de cicatrização, por isso, foi o escolhido de eleição neste estudo ${ }^{13}$.

Para profissionais de saúde, incluindo o fisioterapeuta, colaborar com um recurso que preserve as funções fisiológicas e a qualidade do tecido neoformado é de grande importância, uma vez que as complicações cicatriciais vão além do componente estético, promovem retrações teciduais, diminuição do movimento, dor, alteração de postura e, por fim, redução na qualidade de vida.

Devido à similaridade clínica, laboratorial e histopatológica com 'feridas em humanos', modelos experimentais de lesões cutâneas induzidas em ratos têm sido utilizados largamente por pesquisadores no mundo inteiro. Portanto, o presente estudo teve como objetivo verificar a efetividade do laser $\mathrm{HeNe}$, comparado ao controle sem intervenção, na cicatrização de feridas em animais experimentais (ratos).

\section{METODOLOGIA}

Foi realizada uma revisão sistemática, constituída por estudos experimentais com animais. Os estudos foram previamente selecionados seguindo os critérios de inclusão: utilização do laser $\mathrm{HeNe}$ para o tratamento de feridas agudas comparado a nenhum outro tratamento, que estudaram ratos adultos saudáveis, com lesões induzidas por bisturi. Foram excluídos os estudos que utilizaram: ratos que fossem modelos experimentais de patologias como diabetes e hipertensão; grupo tratado com alguma droga anti-inflamatória esteroidal e não esteroidal; que compararam o laser com qualquer outro tratamento e que foram realizados com feridas crônicas e/ou infectadas.

Considerou-se como desfecho primário o relato da aceleração do processo de reepitelização da ferida e como desfechos secundários: a intensidade da resposta inflamatória e a formação e organização de fibras colágenas geradas pela irradiação do laser na ferida, análise realizada por meio de cortes histológicos das cicatrizes, 
por microscopia eletrônica. Para reconhecimento do padrão inflamatório, os estudos realizaram avaliação morfológica geral da cicatriz por meio da coloração com hematoxilina-eosina (HE) e visualização em microscopia de luz branca.

A estratégia de busca utilizada foi realizada por dois avaliadores independentes, que constava de procura sistemática por artigos publicados em revistas indexadas nas bases de dados PubMed/MEDLINE, LILACS e SciELO. As palavras-chave utilizadas foram: cicatrização de feridas e colágeno (de acordo com o MeSH e $\mathrm{DeCS}$ ), além dos unitermos laser $\mathrm{HeNe}$, reparação da pele e seus equivalentes em inglês e espanhol. As combinações entre tais palavras foram realizadas em cada base de dados supracitadas, utilizando o operador booleano and, sem restrição linguística.

A princípio, os títulos e os resumos identificados pela busca eletrônica foram avaliados independentemente por dois revisores na tela do computador para selecionar os estudos que alcançaram os critérios de elegibilidade. Os estudos potencialmente relevantes, que geraram dúvidas, foram retidos para uma análise posterior.

A avaliação do risco de vieses dos estudos individuais também foi realizada por dois avaliadores de forma independente. Alguns dos critérios publicados para estudos com animais experimentais ${ }^{14}$ foram adaptados e utilizados para o presente estudo: declaração de controle de temperatura; cegamento para indução da lesão; cegamento para avaliação do desfecho; modelo animal apropriado; cálculo de amostra; concordância com regulamentos de bem-estar animal; declaração de potenciais conflitos de interesse e randomização. Para cada estudo foi atribuído um escore de até 8 , sendo a média do grupo calculada. A qualidade do estudo foi considerada baixa quando a pontuação alcançou um valor $\leq 3$.

Todas as divergências encontradas no processo de seleção e na análise dos artigos foram discutidas com um terceiro avaliador. Finalizada a coleta dos dados, verificou-se a possibilidade da construção de uma metanálise. No entanto, esta não foi possível devido à impossibilidade de comparação entre as metodologias dos estudos inclusos.

\section{RESULTADOS}

Entre 101 dos artigos inicialmente selecionados pela busca eletrônica nas bases de dados, 67 foram excluídos pelo título por não atenderem aos critérios de inclusão. Dos 34 estudos retidos, 18 foram eliminados por duplicidade. Foram selecionados 16 estudos para uma análise mais criteriosa por meio do resumo, sendo 12 deles removidos. Os quatro artigos restantes foram avaliados a partir da leitura do texto, sendo que apenas um foi excluído por não se tratar do mesmo método de avaliação da quantidade de fibras colágenas. Um total de três estudos foi incluso: Busnardo et al. ${ }^{15}$, Yasukawa et al. ${ }^{16} \mathrm{e}$ Araújo et al. ${ }^{13}$ (Figura 1).

O resultado da avaliação da qualidade metodológica dos estudos inclusos está descrito na Tabela 1 .

Os estudos de Araújo et al., Yasukawa et al. e Busnardo et al. ${ }^{13,15,16}$ incluíram ratos Wistar adultos submetidos à indução anestésica para realização de tricotomia da região dorsal, seguida de antissepsia local e incisão longitudinal no dorso, com espessura compreendida entre a pele e a tela subcutânea. Araújo et al. ${ }^{13}$ realizaram duas incisões com $6 \mathrm{~mm}$ de diâmetro cada. No estudo de Busnardo et al. ${ }^{15}$, realizou-se apenas uma incisão com aproximadamente $4 \mathrm{~cm}$, nela foram aplicados quatro pontos separados por $1 \mathrm{~cm}$ de distância entre eles. Yasukawua et al. ${ }^{16}$ realizaram 3 incisões de $15 \mathrm{~mm}$ cada.

Não houve homogeneidade nos estudos quanto à quantidade de grupos experimentais e também do número de animais por grupo. Araújo et al. ${ }^{13}$ incluíram 30 ratos, nos quais foram realizadas duas lesões, sendo uma irradiada e outra não, funcionando cada animal como seu próprio controle. No estudo de Busnardo et al. ${ }^{15}$, foram incluídos 60 de tais animais, os quais, após a recuperação anestésica, foram devidamente marcados e distribuídos aleatoriamente em dois grupos, Controle e Experimental, compostos por 30 animais cada. Por fim, o estudo de Yasukawa et al. ${ }^{16}$, que incluiu 55 animais divididos em 11 grupos, sendo 1 Controle e 10 Experimentais (Tabela 2).

Os autores iniciaram a terapia com laser 24 horas após a realização da lesão, porém não foram unânimes em relação aos parâmetros empregados, à frequência de

Tabela 1. Características dos estudos inclusos

\begin{tabular}{|c|c|c|c|c|c|c|c|c|c|c|}
\hline Publicação & Ano & 1 & 2 & 3 & 4 & 5 & 6 & 7 & 8 & Escore \\
\hline Busnardo et al. ${ }^{15}$ (Brasil) & 2010 & $x$ & $x$ & & $x$ & & $x$ & & & 4 \\
\hline Araújo et al. ${ }^{13}$ (Brasil) & 2007 & $x$ & & & $x$ & & $x$ & & & 3 \\
\hline Yasukawa et al.16 (Japão) & 2007 & $x$ & & & $x$ & & $x$ & & & 3 \\
\hline
\end{tabular}

1: declaração de controle de temperatura; 2: cegamento para indução da lesão; 3: cegamento para avaliação do desfecho; 4: modelo animal apropriado; 5: cálculo de amostra; 6: concordância com regulamentos de bem-estar animal; 7: declaração de potenciais conflitos de interesse; 8: randomização 
aplicação do laser, assim como ao tempo para a realização da análise histológica (Tabela 2).

Araújo et al. ${ }^{13}$, em seu estudo, utilizaram uma potência contínua $(\mathrm{PC})$ de $10 \mathrm{~mW}$ e densidade de energia (DE) de $1 \mathrm{~J} / \mathrm{cm}^{2}$ durante três minutos (tempo calculado de acordo com a área) para o Grupo Experimental (GE). O grupo teve uma frequência de aplicação no $1^{\circ}$, $5^{\circ}, 8^{\circ}, 12^{\circ}$ e $15^{\circ}$ dias pós-operatórios (DPOs). Os animais foram sacrificados no $8^{\circ}, 12^{\circ}$ e $22^{\circ}$ DPOs para realização da análise histológica.

No estudo de Busnardo et al. ${ }^{15}$, utilizou-se o laser com PC de $5 \mathrm{~mW}$ e DE de $4 \mathrm{~J} / \mathrm{cm}^{2}$ sobre uma área de $3 \mathrm{~cm}^{2}$, resultando em 12 segundos. O GE foi dividido em três subgrupos com o mesmo número de animais:
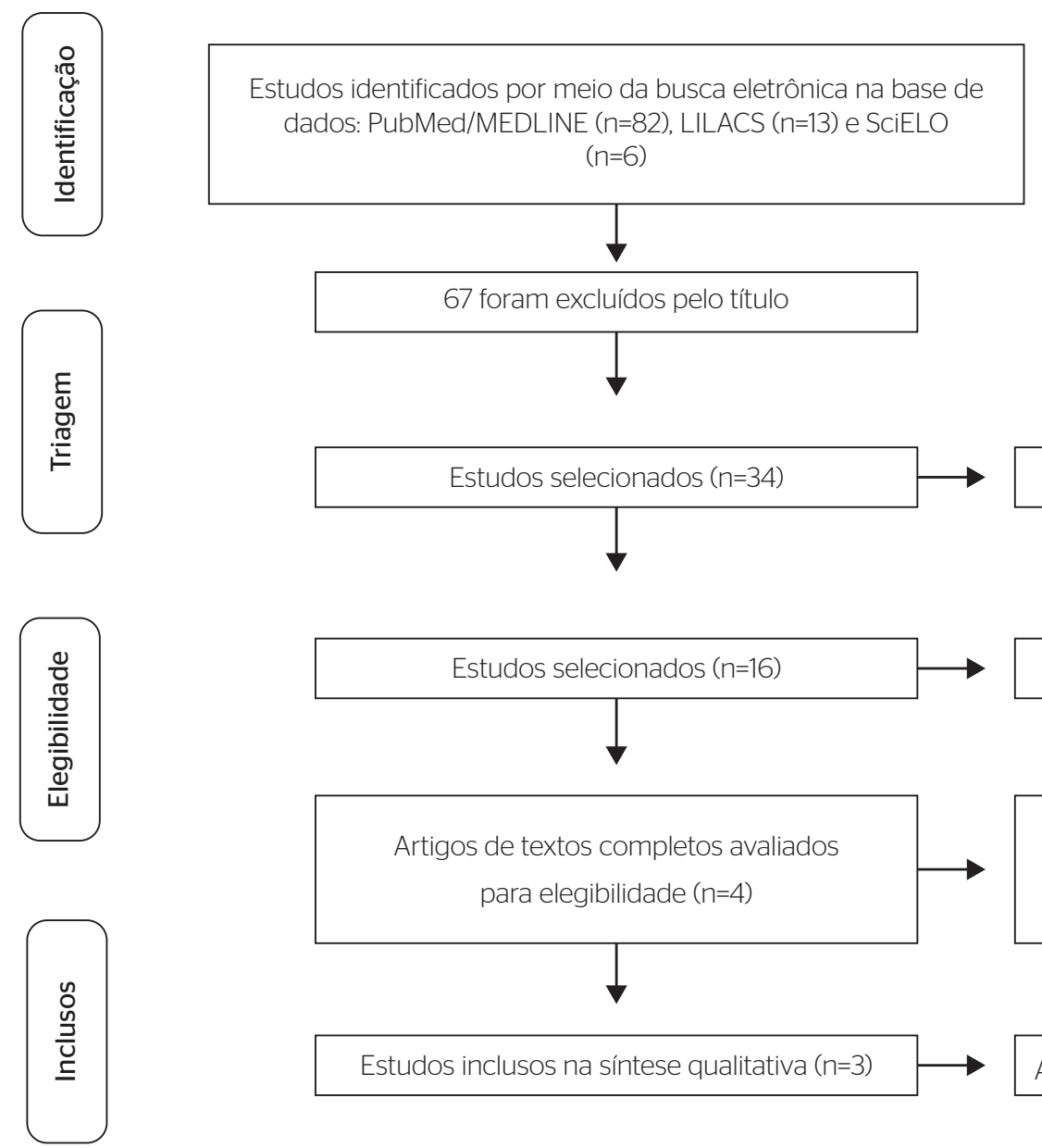

Estudos excluídos por duplicidade ( $n=18)$

Figura 1. Busca e seleção dos estudos para a revisão sistemática de acordo com Preferred Reported Items for Systematic Review and Meta-Analyses (PRISMA)

Tabela 2. Características do estudo, parâmetros e desfechos secundários

\begin{tabular}{|c|c|c|c|c|c|}
\hline Autor & Mascaramento & População & $\begin{array}{l}\text { Intervenção: } \\
\text { parâmetros }\end{array}$ & $\begin{array}{l}\text { Resultados: } \\
\text { resposta inflamatória }\end{array}$ & $\begin{array}{l}\text { Resultados: } \\
\text { fibras colágenas }\end{array}$ \\
\hline $\begin{array}{l}\text { Busnardo et al. } \\
\text { (Brasil) }\end{array}$ & Não citado & $\begin{array}{c}60 \text { ratos }-G C \\
(n=30) \text { e } G E(n=30)\end{array}$ & $\begin{array}{c}P C=5 \mathrm{~mW} \\
D E=4 \mathrm{~J} / \mathrm{cm}^{2} \\
T=12 \text { segundos }\end{array}$ & $\begin{array}{l}\text { No GE houve redução } \\
\text { mais rápida do número } \\
\text { de leucócitos. }\end{array}$ & $\begin{array}{l}\text { Mostrou maior densidade na } \\
\text { avaliação do terceiro dia, não } \\
\text { havendo diferenças significantes } \\
\text { entre o } 70 \text { e } 140 \text { dias. }\end{array}$ \\
\hline Araújo et al. ${ }^{13}$ (Brasil) & Não citado & 30 ratos & $\begin{array}{l}P C=10 \mathrm{~mW} \\
D E=1 \mathrm{~J} / \mathrm{cm}^{2} \\
T=3 \text { minutos }\end{array}$ & $\begin{array}{l}\text { A intensidade da reposta } \\
\text { inflamatória no GE foi } \\
\text { menor do que no GC. }\end{array}$ & $\begin{array}{l}\text { Após o } 150 \text { dia houve uma maior } \\
\text { e melhor organização das fibras } \\
\text { colágenas. }\end{array}$ \\
\hline $\begin{array}{l}\text { Yasukawa et al.16 } \\
\text { (Japão) }\end{array}$ & Não citado & $\begin{array}{c}55 \text { ratos }- \text { GC }(n=5) \\
\quad \text { e GE }(n=50)\end{array}$ & $\begin{array}{c}P C=8,5 \text { ou } 17 \mathrm{~mW} \\
D E=2,09 \text { ou } 4,21 \mathrm{~J} / \mathrm{cm}^{2} \\
T=15 \text { segundos }\end{array}$ & $\begin{array}{l}\text { No GE irradiado com } \\
\text { PC=8,5 mW, houve } \\
\text { leve inflamação com } \\
\text { infiltração de linfócitos } \\
\text { na epiderme. No GE } \\
\text { irradiado com PC=17 mW, } \\
\text { não houve inflamação. }\end{array}$ & $\begin{array}{l}\text { No GE com PC=8,5 mW, a formação } \\
\text { de fibras colágenas não foi } \\
\text { significante. No GE com PC=17 mW, } \\
\text { foi evidenciada formação de fibras } \\
\text { colágenas no centro da lesão. }\end{array}$ \\
\hline
\end{tabular}


um deles recebeu aplicação por três dias, o outro por sete e o último por 14. Os animais foram submetidos à eutanásia em cada um dos tempos e foram ressecados segmentos de pele da cicatriz para análise.

Yasukawa et al. ${ }^{16}$ realizaram um estudo, em que os dez subgrupos do GE receberam PC de 8,5 ou $17 \mathrm{~mW}$ e DE de 2,09 ou $4,21 \mathrm{~J} / \mathrm{cm}^{2}$, respectivamente, por 15 segundos de laser $\mathrm{HeNe}$. As frequências de aplicação foram realizadas em cinco períodos distintos: diariamente (primeiro ao sexto DPO); de forma intercalada (primeiro, terceiro e quinto DPO); apenas no primeiro; apenas no terceiro e apenas no quinto DPO. No sétimo, os animais foram sacrificados para realização da retirada da pele a ser estudada.

O estudo de Araújo et al. ${ }^{13}$ relata que, no oitavo DPO, a área irradiada do GE, apresentava-se completamente reepitelizada e com menor atividade inflamatória, comparada ao mesmo período do Grupo Controle (GC), no qual a atividade inflamatória apresentava-se bastante intensa, caracterizada pela presença de um grande número de leucócitos. Yasukawa et al. ${ }^{16}$, em seu experimento, utilizaram duas potências de irradiações em frequências de aplicações diferentes nas lesões. No quinto dia, observou-se que o grupo irradiado com PC de $8,5 \mathrm{~mW}$ apresentou leve intensidade no processo inflamatório com infiltração de linfócitos imediatamente abaixo da epiderme. No grupo que foi irradiado com $\mathrm{PC}$ de $17 \mathrm{~mW}$, de forma intermitente, não foi observada atividade inflamatória. Segundo Busnardo et al. ${ }^{15}$, no GE, houve redução mais rápida no número de leucócitos do que no GC.

Os estudos relatam que a terapia por laser de baixa potência contribui para o aumento na quantidade de colágeno e fibras elásticas na fase inicial do processo de cicatrização. Araújo et al. ${ }^{13}$ observaram em seu estudo que o $\mathrm{GE}$ a partir do $15^{\circ} \mathrm{DPO}$ apresentava maior organização das fibras colágenas e, ainda, que, no 22 DPO, apresentou um aumento na quantidade de fibroblastos. No quinto dia, Yasukawa et al. ${ }^{16}$ observaram que, no grupo irradiado com PC de $8,5 \mathrm{~mW}$, a formação de colágeno não foi significante. No grupo que foi irradiado com PC de $17 \mathrm{~mW}$ de forma intermitente, porém, houve formação de colágeno no centro da cicatriz. No estudo de Busnardo et al. ${ }^{15}$ foi observado, no terceiro DPO, um aumento da síntese de colágeno tipo III, assim como um incremento na porcentagem de colágeno total, porém sem diferenças significantes nas avaliações do $7^{\circ}$ e $14^{\circ}$ DPOs.

\section{DISCUSSÃO}

De acordo com os resultados da presente revisão sistemática, a terapia com laser $\mathrm{HeNe}$ apresentou boa resposta em relação ao reparo tecidual. No entanto, devem ser analisados de maneira criteriosa, uma vez que os estudos disponíveis e inclusos não possuíam o rigor metodológico adequado, podendo comprometer a análise final dos resultados e, consequentemente, a recomendação dessa prática. Além disso, a presença de heterogeneidade, principalmente em relação aos parâmetros adotados entre os estudos, dificulta a avaliação fidedigna da real eficácia desta terapia ${ }^{13,15,16}$. Estudos prévios apontam para a eficiência da terapia a laser na aceleração do processo de reepitelização, relacionando-a à redução da atividade inflamatória e ao aumento da síntese de colágeno ${ }^{2,3,17,18}$.

Os efeitos biológicos do laser de baixa potência têm sua função baseada em um processo fotobiológico, que consiste na absorção molecular de energia luminosa, depositada e se transforma em energia vital, produzindo, então, efeitos primários, como estimulação de linfócitos, ativação de mastócitos e aumento na produção de adenosina trifosfato (ATP) mitocondrial e terapêuticos gerais, os quais promovem ações de natureza analgésica, anti-inflamatória e cicatrizante , $^{3,719-21}$.

Não foi observada uniformidade entre os estudos quanto aos valores dos parâmetros utilizados. Houve variação da $\mathrm{DE}$ e do tempo de aplicação. Com relação à potência, todos os estudos a utilizaram de forma contínua, embora com valores diferentes, que variaram entre 5 e $17 \mathrm{~mW}$. O tempo de aplicação por ponto da ferida oscilou entre 12 segundos e três minutos, enquanto a $\mathrm{DE}$ variou entre 1 e $4,21 \mathrm{~J} / \mathrm{cm}^{2}{ }^{13,15,16}$. Não se encontra consenso na literatura em relação aos protocolos de utilização da terapia a laser, porém alguns autores sugerem que os valores de DE utilizados para cicatrização de feridas devem variar entre 3 e $6 \mathrm{~J} / \mathrm{cm}^{28}$.

Foi evidenciada discordância entre os estudos quanto à periodicidade e à frequência de utilização do recurso terapêutico nos grupos experimentais, sendo a aplicação efetuada entre o $1^{\circ}$ e o $15^{\circ}$ dia após a incisão cirúrgica ${ }^{13,15,16}$.

Em relação à qualidade metodológica, os resultados da presente revisão sistemática demonstram que os estudos obtiveram pontuações variando entre três e quatro. Em todos os estudos, os animais foram acondicionados em gaiolas com as mesmas condições ambientais e de acesso à alimentação e à água. Os experimentos foram conduzidos de acordo com protocolos aprovados pelos respectivos Comitês de Ética em Pesquisa Animal. 
Dentre eles, apenas no estudo de Busnardo et al. ${ }^{15}$ observou-se a descrição da realização do mascaramento para indução da lesão. Nenhum dos estudos referiu realização de randomização, ferramenta importante que minimiza o viés de seleção e, desse modo, evita interferência direta no resultado do tratamento, que pode alcançar um valor superestimado em torno de $40 \%$ na ausência de sua aplicação ${ }^{22-24}$. Quanto ao controle relativo do mascaramento para análise dos dados, nenhum estudo descreveu se houve cegamento para avaliação dos dados coletados ou durante a realização da análise estatística. $\mathrm{O}$ mascaramento constitui-se em uma importante ferramenta para o desenho de estudo experimental, tendo como objetivo principal minimizar o viés de aferição e de avaliação dos desfechos, evitando uma interferência direta do pesquisador no resultado do tratamento ${ }^{22,24}$.

Mais estudos clínicos devem ser realizados, com o objetivo de avaliar a aplicação do laser de baixa intensidade, uma vez que, na literatura pertinente, há uma diversidade na metodologia empregada, tais como diferenças no comprimento de onda, dosimetrias, tipos de estudo e desenhos experimentais ${ }^{20}$.

Apesar de ser um recurso utilizado há algumas décadas, ainda não existe consenso sobre a padronização das variáveis físicas. Além disso, a maioria dos estudos apresenta falhas metodológicas pela não especificação dos parâmetros adotados em seus experimentos. Nesse sentido, Damante et al. ${ }^{25}$ sugeriram, em seu estudo, um checklist relatando quais seriam os parâmetros que deveriam ser relatados nos estudos com tal terapia quanto ao laser (comprimento de onda, potência, modo, meio ativo e calibração); à dose (DE, área tratada ou de ponta ativa, tempo de aplicação e número de pontos tratados); à técnica de aplicação (local e modo) e ao tipo de tratamento (frequência e número).

\section{CONCLUSÕES}

De acordo com a presente revisão sistemática, a terapia com laser $\mathrm{HeNe}$ pode apresentar resposta satisfatória na reepitelização das feridas induzidas cirurgicamente em ratos, principalmente pela redução na intensidade da resposta inflamatória e pelo aumento na deposição de colágeno total.

Entretanto, observa-se a necessidade de estudos que abordem o uso do laser no processo de reparo tecidual em ratos, com alto rigor metodológico, além de ensaios clínicos controlados em humanos, principalmente em relação aos parâmetros adotados. Tais estudos devem ter o intuito de estabelecer um protocolo-padrão para se ter uma avaliação de desfecho de forma mais fidedigna e uma indicação segura, baseada em evidência científica, de seu uso na prática clínica.

\section{AGRADECIMENTOS}

À Viviane Patriota Alexandre por sua importante contribuição na elaboração deste manuscrito.

\section{REFERÊNCIAS}

1. Gonçalves WL, Souza FM, Conti CL, Cirqueira JP, Rocha WA, Pires JGP, et al. Influence of He-Ne laser therapy on the dynamics of wound healing in mice treated with anti-inflammatory drugs. Braz J Med Biol Res. 2007;40(6):877-84

2. Carvalho PT, Mazzer N, Reis FA, Belchior AC, Silva IS. Analysis of the influence of low-power HeNe laser on the healing of skin wounds in diabetic and non - diabetic rats. Acta Cir Bras. 2006;21(3):177-83.

3. Carvalho PT, Silva IS, Reis FA, Perreira DM, Aydos RD. Influence of ingaalp laser (660nm) on the healing of skin wounds in diabetic rats. Acta Cir Bras. 2010;25(1):71-9.

4. Nascimento DG, Fernandes CAM, Sartoretto JL, Bruschi LC, Cuman RKN, Silva FP. Efeitos da cicatrização com laser HeNe 632.8 nm sobre a cicatrização de feridas em ratos. Cienc Cuid Saúde. 2006;5:229-35.

5. Júnior AM, Vieira BJ, Andrade LC, Aarestrup FM. Effects of low-level laser therapy on the progress of wound healing in humans: the contribution of in vitro and in vivo experimental studies. J Vasc Bras. 2007;6:258-66

6. Simões NP, Almeida MF, Nohama P. Estudo comparativo entre laser He-Ne e a estimulação elétrica microamperada na cicatrização de pele de ratos. Rev Kinesia. 2009;:19-30.

7. Pugliese LS, Medrado AP, Reis SRA, Andrade ZA. The influence of low - level laser therapy on biomodulation of collagen and elastic fibers. Pesqui Odontol Bras. 2003;17(4):307-13.

8. Low J, Reed A. Tratamento com radiações eletromagnéticas. In: Robertson V, Ward A, Low J, Reed A. Eletroterapia explicada: princípios e prática. 3 ed. São Paulo: Editora Manole; 2001. p. 385-98.

9. Silveira PC, Silva LA, Tuon T, Freitas TP, Streck EL, Pinho RA. Efeitos da laserterapia de baixa potência na resposta oxidativa epidérmica induzida pela cicatrização de feridas. Rev Bras Fisioter. 2009;13:281-7.

10. Inoe AP, Zafanelli CC, Rossato RM, Leme MC, Sanches AW, Araujo CV, et al. Avaliação morfológica do efeito do laser de baixa potência HeNe em feridas cutâneas de coelhos. Arq Cienc Vet Zool. 2008;11:27-30.

11. Pinto NC, Pereira MH, Stolf NA, Chavantes MC. Laser de baixa intensidade em deiscência aguda de safenectomia: proposta terapêutica. Rev Bras Cir Cardiovasc. 2009;24:88-91.

12. Silva EM, Gomes SP, Ulbrich LM, Giovanini AF. Avaliação histológica da laserterapia de baixa intensidade na cicatrização de tecidos epitelial, conjuntivo e ósseo: estudo experimental de ratos. RSBO. 2007:4:29-35 
13. Araújo CE, Ribeiro MS, Favaro R, Zezell DM, Zorn TM. Ultrastructural and autoradiographical analysis show a faster skin repair in He-Ne laser-treated wounds. J Photochem Photobiol B Biol. 2007;86(2):87-96.

14. Macleod MR, O'Collins T, Howells DW, Donnan GA. Pooling of Animal Experimental Data Reveals Influence of Study Design and Publication Bias. Stroke. 2004;35(5):1203-8.

15. Busnardo VL, Biondo-Simões ML. Effects of low-level helium-neon laser on induced wound healing in rats. Rev Bras Fisioter. 2010;14(1):45-51.

16. Yasukawa A, Ohrui H, Koyama Y, Nagai M, Takakuda K. The effect of low reactive - level laser therapy (LLLT) with helium-neon laser on operative wound healing in a rat model. J Vet Med Sci. 2007:69(8):799-806.

17. Silveira PC, Silva LA, Freitas TP, Latini A, Pinho RA. Effects of lowpower laser irradiation (LPLI) at different wavelengths and doses on oxidative stress and fibrogenesis parameters in an animal model of wound healing. Lasers Med Sci. 2011;26(1):125-31.

18. Rocha Júnior AM, Oliveira RG, Farias RE, Andrade LCR, Aarestrup FM. Modulação da proliferação fibroblástica e da resposta inflamatória pela terapia a laser de baixa intensidade no processo de reparo tecidual. An Bras Dermatol. 2006;81:150-6.
19. Lins RD, Dantas EM, Lucena KC, Catao MH, Granville AF, Neto LG. Efeitos bioestimulantes do laser de baixa potência no processo de reparo. An Bras Dermatol. 2010;85:849-55.

20. Barros FC, Antunes SA, Figueredo CM, Fischer RG. Laser de baixa intensidade na cicatrização periodontal. Rev Cienc Med Biol. 2008;7:85-9.

21. Melo VA, Anjo DC, Júnior RA, Melo DB, Carvalho FU. Effect of low level laser on sutured wound healing in rats. Acta Cir Bras. 2011;26(2):129-34.

22. Cummings SR, Grady D, Hulley SB. Delineando um experiment: ensaios clínicos I. In: Hulley SB, Cummings SR, Browner WS, Grady D, Hearst N. Newman TB. Delineando a pesquisa clínica: uma abordagem epidemiológica. 2 ed. São Paulo: Artmed; 1998. p. 165-79.

23. Montori VM, Guyatt GH. Intention-to-treat principle. CMAJ. 2001:165(10):1339-41.

24. Schulz KF. Unbiased research and the human spirit: the challenges of randomized controlled trials. CMAJ. 1995;153(6):783-6.

25. Damante CA, Marques MM, De Micheli G. Terapia com laser em baixa intensidade na cicatrização de feridas - revisão de literatura. RFO. 2008:13:88-93. 